\title{
b-chromatic number of cacti $^{2}$
}

\author{
Victor Campos ${ }^{c, 1}$ Cláudia Linhares Sales ${ }^{c, 1}$ \\ Frédéric Maffray ${ }^{\mathrm{b}, 1}$ Ana Silva ${ }^{\mathrm{a}, 1}$ \\ a Laboratoire G-SCOP, Université Joseph Fourier, Grenoble, France \\ b CNRS, Laboratoire G-SCOP, Grenoble, France \\ c Departamento de Computação, Universidade Federal do Ceará(UFC), Brazil
}

\begin{abstract}
A b-colouring of a graph $G$ is a proper colouring of $G$ such that each colour contains a vertex that is adjacent to all other colours and the b-chromatic number $\chi_{b}(G)$ is the maximum number of colours used in a b-colouring of $G$. If $m(G)$ is the largest integer $k$ such that $G$ has at least $k$ vertices with degree at least $k-1$, then we know that $\chi_{b}(G) \leq m(G)$. Irving and Manlove [1] prove that, if $T$ is a tree, then the b-chromatic number of $T$ is at least $m(T)-1$. In this paper, we prove that, if $G$ is a connected cactus and $m(G) \geq 7$, then the b-chromatic number of $G$ is at least $m(G)-1$.
\end{abstract}

Keywords: Graph, complexity, b-colouring, cactus

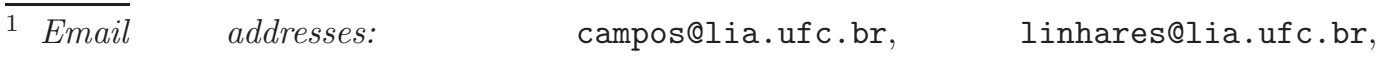
Frederic.Maffray@g-scop.inpg.fr, Ana.Silva@g-scop.inpg.fr

2 Partially supported by CAPES,CNPq/Brazil and CNRS/France
} 


\section{Introduction}

Let $G$ be a simple graph. A proper coloring of $G$ is an assignment of colors to the vertices of $G$ such that no two adjacent vertices have the same color. In a proper coloring, the color class of a color $c$ is the set of vertices of $G$ colored with color $c$. The chromatic number of $G$ is the minimum integer $\chi(G)$ such that $G$ has a proper coloring with $\chi(G)$ colors. Suppose that we have a proper coloring of $G$ and a color class $C$ such that every vertex in $C$ is not adjacent to at least one other color class. We can change the color of the vertices in $C$, obtaining a proper coloring that uses less colors than before. However, as one can expect, we cannot apply this heuristic iteratively until we reach the chromatic number of $G$, since the coloring problem is $\mathcal{N} \mathcal{P}$-hard. It was this idea that made Irving and Manlove introduce the notion of b-coloring in [1]. Intuitively, a b-coloring is a proper coloring that cannot be improved by the described heuristic and the b-chromatic number $\chi_{b}(G)$ of $G$ measures the worst possible such coloring. More formally:

Definition 1.1 A vertex $u$ in color class $C$ is said to be a b-vertex if $u$ has at least one neighbor in each color class other than $C$.

Definition 1.2 A b-coloring of a graph $G$ is a proper coloring of $G$ such that each color class contains at least one b-vertex.

Definition 1.3 The b-chromatic number of $G$ is the largest integer $k$ such that $G$ has a b-coloring with $k$ colors. We denote it by $\chi_{b}(G)$.

Naturally, we have that a proper coloring of $G$ with $\chi(G)$ colors is a bcoloring of $G$, since it cannot be improved. So, $\chi(G) \leq \chi_{b}(G)$. For an upper bound, note that if $G$ has a b-coloring with $k$ colors, then $G$ has at least $k$ vertices with degree at least $k-1$ (the b-vertices). So, if $m(G)$ is the largest integer such that $G$ has at least $m(G)$ vertices with degree at least $m(G)-1$, we know that $G$ cannot have a b-coloring with more than $m(G)$ colors, i.e., $\chi_{b}(G) \leq m(G)$. This upper bound was introduced by Irving and Manlove in [1], where they also showed that the difference between $\chi_{b}(G)$ and $m(G)$ can be arbitrarily large for a general graph and that it is at most one for trees. In addition, the problem was proved to be $\mathcal{N} \mathcal{P}$-hard [1], even when restricted to bipartite graphs [2].

We say that $G$ is a cactus if $G$ does not contain two cycles that share an edge. In this article, we prove that, if $G$ is a connected cactus and $m(G) \geq 7$, then the difference between $\chi_{b}(G)$ and $m(G)$ is at most one and we can obtain $\chi_{b}(G)$ in polynomial time. 
We say that $G$ is $m$-defective if $m(G)=m$ and $\chi_{b}(G)<m$. Additionally, $G$ is minimal $m$-defective if $G$ is $m$-defective and any proper subgraph of $G$ is not $m$-defective.

The general idea for our main result is as follows. We present the class of pivoted cacti and show that every pivoted cactus $G$ is $m(G)$-defective. Then, we show that $\chi_{b}(G)=m(G)-1$, for every pivoted cactus $G$. Finally, we prove that a minimal $m(G)$-defective cactus is pivoted. To do so, first we describe the structure of minimal $m(G)$-defective cacti. We say that a subgraph $H$ of $G$ is a b-kernel of $G$ if $m(H)=m(G)$ and $\chi_{b}(H)=m(G)$ implies $\chi_{b}(G)=m(G)$. If $G$ is not a pivoted cactus, we find a special b-kernel $H_{G}$ of $G$ and we either show how to b-color $H_{G}$ with $m(G)$ colors or show that $H_{G}$ contains no minimal $m(G)$-defective cactus as a subgraph. In any case, this implies that $\chi_{b}\left(H_{G}\right)=m(G)$ and, by the definition of a b-kernel, $\chi_{b}(G)=m(G)$. This proves that if $G$ is not a pivoted cactus, then it is not $m(G)$-defective. More details on this general idea are provided in the remainder of this paper.

\section{Pivoted Cacti}

In this section, we define the class of pivoted cacti and show that if $G$ is a pivoted cactus, then $\chi_{b}(G)=m(G)-1$. This implies that every pivoted cactus $G$ is $m(G)$-defective.

Let $G=(V, E)$ be a connected cactus. We say that a vertex $v$ in $V$ is a dense vertex if $d(v) \geq m(G)-1$. Let $M(G)$ denote the set of dense vertices of $G$.

Let $V^{\prime}$ be any subset of $m(G)$ vertices of $M(G)$. Let $u \in V \backslash V^{\prime}$ and $v \in V^{\prime}$. If $u$ and $v$ are adjacent or have a common neighbor $w$ in $V^{\prime}$ with $d(w)=m(G)-1$, then we say that $v$ is reachable from $u$ within $V^{\prime}$. Note that if $u \in V \backslash V^{\prime}$ reaches every vertex of $V^{\prime}$, then there is no b-colouring of $G$ with $V^{\prime}$ as the set of b-vertices. Observe that it also happens if we have a pair of vertices $u, v \in V \backslash V^{\prime}$ that reaches every vertex of $V^{\prime}$ but one, say $w$ : we could colour $u$ or $v$ with the colour of $w$, but the other one cannot be coloured without repeating some colour in the neighborhood of a vertex of $V^{\prime}$ with degree $m(G)-1$. So, we say that $V^{\prime}$ encircles vertex $u$ in $V \backslash V^{\prime}$ if every vertex $v$ in $V^{\prime}$ is reachable from $u$ within $V^{\prime}$. Below, we describe the situations where we have a pair that prevent $V^{\prime}$ from being a set of b-vertices of some b-colouring of $G$. We say that $V^{\prime}$ encircles the pair $x, y \in V \backslash V^{\prime}$ if it does not encircle $x$ or $y$ and if one of the following occurs:

(E1) There are $V^{\prime \prime} \subset V^{\prime}$ and $u, v \in V^{\prime \prime}$ such that $\left|V^{\prime \prime}\right|=m(G)-1,\langle x, u, y, v\rangle$ 
is a cycle and:

(a) $d(u)=d(v)=m(G)-1$ and every $w \in V^{\prime \prime} \backslash\{u, v\}$ is adjacent to $u$ or $v$; or

(b) $d(u)=m(G)-1$ and every $w \in V^{\prime \prime} \backslash\{u, v\}$ is adjacent to $u$; or

(c) $d(u)=m(G), d(v)=m(G)-1$ and every $w \in V^{\prime} \backslash\{u, v\}$ is adjacent to $u$ or $v$; or

(d) $d(u)=m(G)$ and every $w \in V^{\prime} \backslash\{u, v\}$ is adjacent to $u$.

(E2) There are $V^{\prime \prime} \subseteq V^{\prime}$ and $u, v, w \in V^{\prime \prime}$ such that $\left|V^{\prime \prime}\right| \geq m(G)-1$, $\langle x, u, v, y, w\rangle$ is a cycle, $d(u)=d(v)=m(G)-1$, every $w^{\prime} \in V^{\prime \prime} \backslash\{u, v, w\}$ is adjacent to $w$, and

(a) $V^{\prime \prime}=V^{\prime}$ and $d(w)=m(G)$; or

(b) $V^{\prime \prime} \subset V^{\prime}$ and $d(w)=m(G)-1$.

Let $V^{\prime}$ be a subset of $m(G)$ vertices of $M(G)$. We say that $V^{\prime}$ is a good set if it does not encircle any vertex or pair of vertices and every $u \in V \backslash V^{\prime}$ with degree at least $m(G)$ is either adjacent to some vertex in $V^{\prime}$ with degree $m(G)-1$ or is within a path between two vertices of $V^{\prime}$ of length at most three, whose internal vertices are not in $V^{\prime}$. If $G$ does not have a good set, we say that $G$ is a pivoted cactus.

The following lemma shows the possible number of encircled vertices or encircled pairs.

Lemma 2.1 Let $V^{\prime}$ be any set of $m(G)$ dense vertices. If $m(G) \geq 7$, then $V^{\prime}$ encircles at most two vertices, or at most one pair of vertices.

By using the structural properties presented in Lemma 2.1, we can prove that if $|M(G)|=m(G)$ and situation E1 or E2 occurs (in which case, we know that $\left.\chi_{b}(G)<m(G)\right)$, then we can b-color $G$ with $m(G)-1$ colors. However, the following lemma shows us that there is a situation where $G$ has more than $m(G)$ dense vertices and still cannot be b-colored with $m(G)$ colors.

Lemma 2.2 Let $G$ be a connected cactus with $|M(G)|>m(G), m(G) \geq 7$, and let $V^{\prime}$ be a set of $m(G)+1$ dense vertices of $G$ containing all vertices with degree greater than $m(G)-1$. Then, $G$ does not have a good set if and only if $V^{\prime}=M(G)$ and there are vertices $u, v \in V^{\prime}$ and $w \in V \backslash V^{\prime}$ such that $d(u)=d(v)=m(G)-1,\{u, v, w\}$ forms a triangle in $G$ and every vertex in $V^{\prime}$ is adjacent to $u$ or to $v$.

Again, we can use the structural properties presented in Lemma 2.2 to prove the following lemma by giving a b-coloring of $G$ with $m(G)-1$ colors.

Lemma 2.3 If $G$ is a pivoted connected cactus, then $\chi_{b}(G)=m(G)-1$. 


\section{Non pivoted Cacti}

In this section, we prove that if a cactus $G$ has a good set, then it can be b-coloured with $m(G)$ colours, giving us the desired result. To do so, we first describe the structure of minimal $m$-defective cacti.

Theorem 3.1 If $G$ is a minimal $m(G)$-defective cactus and $m(G) \geq 7$, then $|M(G)|=m(G)$ and $d(v)=m(G)-1$, for all $v \in M(G)$. Furthermore, if $u \in V(G) \backslash M(G)$, then any neighbor of $u$ is a dense vertex of $G$.

Theorem 3.2 If $G$ is a minimal $m(G)$-defective cactus with $m(G) \geq 7$, then either $M(G)$ encircles a vertex or $M(G)$ encircles a pair of vertices as in E1.a or E2.b.

To link minimal $m$-defective cacti with non-pivoted cacti, note that if $G$ has no minimal $m(G)$-defective subgraph, then $G$ is not defective. This implies that $\chi_{b}(G)=m(G)$. We also use the following lemma.

Lemma 3.3 If $G$ is a non-pivoted cactus and $V^{\prime}$ is a good set of $G$, then $G\left[V^{\prime} \cup N\left(V^{\prime}\right)\right]$ is a b-kernel of $G$.

Now, let $G$ be a non-pivoted cactus and let $V^{\prime}$ be a good set of $G$. Consider the b-kernel $H_{G}=G\left[V^{\prime} \cup N\left(V^{\prime}\right)\right]$ of $G$. If $H_{G}$ contains no minimal $m(G)$ defective subgraph, then $H_{G}$ is not $m(G)$-defective itself and we have $\chi_{b}(H)=$ $m(G)$. Since $H_{G}$ is a b-kernel, then $\chi_{b}(G)=m(G)$. On the other hand, if $H_{G}$ has a minimal $m(G)$-defective subgraph, then using the structural properties given by Theorem 3.2 and the fact that $V^{\prime}$ does not encircle a vertex or pair of vertices in $H_{G}$, it is possible to construct a b-colouring of $G$ with $m(G)$ colours. So, the following theorem is true:

Theorem 3.4 If $G$ is a non-pivoted cactus with $m(G) \geq 7$, then $\chi_{b}(G)=$ $m(G)$.

\section{Closing Comments}

Our main result is the following theorem:

Theorem 4.1 If $G$ is a connected cactus with $m(G) \geq 7$, then $m(G)-1 \leq$ $\chi_{b}(G) \leq m(G)$.

In addition, we found an algorithm that gives an optimum b-coloring of a pivoted cactus and an algorithm to find a good set of a non-pivoted cactus. We also have an algorithm to extend a maximum b-coloring of the b-kernel $H_{G}$ 
into a maximum b-coloring of $G$. However, we could not find an algorithm that optimally colors $H_{G}$ and the proof that $\chi_{b}\left(H_{G}\right)=m(G)$ is not constructive. Thus, we can decide in polynomial time the value of $\chi_{b}(G)$, but we cannot always provide such coloring.

\section{References}

[1] Irving, R.W. and Manlove, D.F., The b-chromatic number of a graph, Discrete Applied Mathematics, 91(1999), pages 127-141.

[2] Kratochvil, J. and Tuza, Z. and VOIGT, M. On the b-chromatic number of graphs, Lecture Notes In Computer Science, Vol. 2573, pag. 310-320, 2002. 